\title{
Decreased emotional reactivity after 3-month socio-affective but not attention- or meta-cognitive-based mental training: A randomized, controlled fMRI study
}

Pauline Favre a, 1,2* ${ }^{*}$ Philipp Kanske ${ }^{\text {a, } 3}$, Haakon Engen ${ }^{\text {a, } 4}$ and Tania Singer a, 5

${ }^{\text {a }}$ Max Planck Institute for Human Cognitive and Brain Sciences, Leipzig, Germany

Present addresses:

${ }^{1}$ INSERM U955, team 15 « Translational Neuro-Psychiatry », Créteil, France

${ }^{2}$ Neurospin Neuroimaging Platform, team UNIACT, CEA Paris-Saclay, Gif-sur-Yvette, France

${ }^{3}$ Clinical Psychology and Behavioral Neuroscience, Faculty of Psychology, Technische Universität Dresden, Dresden, Germany

${ }^{4}$ Department of Psychology, University of Oslo, Oslo, Norway

${ }^{5}$ Social Neuroscience Lab, Max Planck Society, Berlin, Germany

* Corresponding author:

Pauline Favre, PhD

Neurospin, CEA Paris-Saclay

91191, Gif-sur-Yvette, France

Phone: +33 (0)1 69082481

Email: pauline@favre-univ.fr 


\begin{abstract}
Meditation-based mental training interventions show great benefits for physical and mental health. However, it remains unclear how different types of mental practice differentially affect emotion processing at both the neuronal and the behavioural level. In the context of the ReSource project, 332 participants underwent an fMRI scan while performing an emotion reactivity task before and after three 3-month training modules cultivating 1) attention and interoceptive awareness (Presence); 2) socio-affective skills, such as compassion (Affect); 3) socio-cognitive skills, such as theory of mind (Perspective). Only the Affect module led to a significant reduction of experienced negative affect when processing images depicting human suffering. This decrease in emotion reactivity was associated with increased activation in cognitive control and emotion-regulation regions such as lateral parietal and prefrontal brain regions. We conclude that socio-affective, but not attention- or meta-cognitive based mental training is specifically efficient to improve emotion regulation capacities when facing adversity.
\end{abstract}

Key-words: meditation, fMRI, emotion, mental training, compassion, mindfulness 


\section{Introduction}

Meditation-based mental training programs have been developed to improve emotion regulation and to decrease symptoms of psychopathology in both clinical and non-clinical populations (Heeren and Philippot, 2011; Kuyken et al., 2016; Piet and Hougaard, 2011; Teasdale et al., 2002). Many of these programs focus on the cultivation of mindfulness (e.g. Mindfulness-Based Stress Reduction (MBSR) and the Mindfulness-Based Cognitive Therapy (MBCT) programs (Kabat-Zinn, 2003; Williams et al., 2014)) or compassion (e.g. the Mindfulness Self-Compassion Program (Neff and Germer, 2013), Compassion-Focused Therapy (Gilbert, 2009) or the Compassion Cultivation Training (Jazaieri et al., 2013)). Studies have shown beneficial effects of such mental training programs on physical health, through stress reduction (Creswell et al., 2014) or improved immune system functions (Pace et al., 2009), as well as on mental health through increasing well-being and decreasing negative emotions (Goldin and Gross, 2010; Gu et al., 2015; Sedlmeier et al., 2012; Wallace and Shapiro, 2006). However, most programs consist of a mixture of different types of mental training practices whose specific effects are still poorly understood, especially on the control of emotional reactivity and its neural correlates. Indeed, there are at least three different domains that might be targeted in these integrative practices to improve emotion regulation: attention, socio-affective and socio-cognitive skills.

The attentional skills were mostly targeted by mindfulness-based interventions (MBI), which aim to develop present-moment attention and interoceptive awareness (Kabat-Zinn, 2003), and were proposed to affect both "bottom-up" and "top-down" emotion regulation mechanisms (Chiesa et al., 2013; Guendelman et al., 2017). Previous functional magnetic resonance imaging (fMRI) studies using MBI have tested various emotion generation and regulation paradigms, such as affect labelling (Hölzel et al., 2013), face processing (Johnson et al., 2014), self-reference (Farb et al., 2007; Goldin et al., 2012) or pain regulation (Zeidan et 
al., 2015) tasks (for review, see Guendelman et al., 2017; Magalhaes et al., 2018; Young et al., 2018). Among them, studies that compared expert vs. novice practitioners and used implicit emotion regulation paradigms (i.e., passive viewing of emotional stimuli) showed conflicting results (Froeliger et al., 2012; Lee et al., 2012; Taylor et al., 2011). Specifically, these studies found both increased and diminished activity of the PFC in expert compared to novice practitioners in response to negative and sad pictures (Froeliger et al., 2012; Lee et al., 2012), as well as no difference when confronted with positive, negative and neutral images (Taylor et al., 2011). In addition, two studies used a longitudinal design and failed to show significant differences between novices practitioners who underwent an MBI vs. a control group (Allen et al., 2012; Desbordes et al., 2012). However, Allen et al. (2012), found that the amount of practice predicted greater activity in fronto-insular regions in a group trained in mindfulness. Another study, using neutral and sad video clips, showed both increased and decreased activity in the PFC and increase activity of the right insula after MBI in comparison to a waitlist control group (Farb et al., 2010). Recent reviews and meta-analyses suggest that MBI more generally increases recruitment of prefrontal regions even in the absence of explicit regulation instruction, thereby allowing automatic control of emotions. They also suggest a strong involvement of the insula which could be associated with better interoceptive awareness, while the modulation of the amygdala's response after mindfulness-meditation training is not yet well understood (Guendelman et al., 2017; Magalhaes et al., 2018; Young et al., 2018).

More recently, there has also been increased interest in studying Compassion-Based Interventions $(\mathrm{CBI})$ that aim to develop social emotions and motivation such as loving kindness and compassion towards the suffering of oneself and of others (Gilbert, 2009, 2017; Jazaieri et al., 2013; Neff and Germer, 2013). Pioneering investigations on the neural correlates of compassion-based meditation practices employed cross-sectional designs to compare the brains of expert long-term meditation practitioners with novice practitioners (Lutz et al., 2008; Lutz 
et al., 2009). By comparing the brain responses of expert practitioners when regulating their emotions using compassion meditation and reappraisal, a "gold standard" emotion regulation technique (Ochsner and Gross, 2005), our group found that both the subjective and neural responses to these techniques were markedly different (Engen and Singer, 2015): First, in terms of subjectively experienced emotion, compassion meditation primarily increased positive emotion while reappraisal strategies decreased negative affect. Second, imaging results revealed that compassion meditation in contrast to reappraisal strategies increased activity in the ventral striatum (VS) and the medial orbito-frontal cortex (OFC), while reappraisal preferentially recruited lateral prefrontal regions. Similarly, with longitudinal mental training designs, Klimecki and colleagues found that participants who trained compassion and lovingkindness meditation reported feeling more positive emotions, which was associated with increased activity in the medial OFC, the nucleus accumbens (NAcc), the VS and midbrain areas (Klimecki et al., 2013; Klimecki et al., 2014). Thus, compassion meditation appears to both increase positive affect and recruit a set of brain areas that are known to be associated with reward, affiliation, positive social feelings and prosocial motivation (Burgdorf and Panksepp, 2006; Carter and Keverne, 2002; McCall and Singer, 2012; O’Doherty, 2004; Schultz, 2006; Singer and Klimecki, 2014) that are distinct from regions most commonly associated with cognitive emotion regulation (Buhle et al., 2014; Ochsner et al., 2002).

Importantly, emotion processing and regulation involves both socio-affective and sociocognitive skills. The recognition that somebody is suffering, for example, is classically associated with the process of empathy, which is defined as the human capacity to share and understand other people's emotions without confusing them with one's own feelings (De Vignemont and Singer, 2006). Functional and structural neuroimaging studies have consistently implicated an extended cerebral network comprising the anterior insula (AI) and anterior cingulate cortex (ACC), as well as lateral prefrontal and parietal areas, such as the DLPFC, 
ventrolateral PFC and supramarginal gyrus (SMG) for empathy (Bzdok et al., 2012; Fan et al., 2011; Kanske et al., 2015; Lamm et al., 2011; Singer et al., 2004). Besides the described socioaffective skills, the understanding of other people's mental states requires socio-cognitive skills, i.e., the capacity to infer thoughts, beliefs and intentions of others, which is termed mentalizing, perspective taking or Theory of Mind (ToM) (Premack and Woodruff, 1978; Saxe and Kanwisher, 2003; Singer, 2012). ToM is thought to be underpinned by a neural network including the temporoparietal junction (TPJ), superior temporal sulcus (STS), temporal pole (TP), medial prefrontal cortex (mPFC) and precuneus/posterior cingulate (PCC) (Bzdok et al., 2012; Frith and Frith, 2005; Kanske et al., 2016; Kanske et al., 2015; Saxe and Kanwisher, 2003; Schurz et al., 2014). The importance of socio-affective and socio-cognitive capacities for individual and societal welfare is incontestable, however the possibility of training these skills and the potential subsequent effects of such a training on emotion processing is not well explored.

A major obstacle to disentangling what effects meditation-based mental training has on emotion processing is that previous randomized-controlled trials (RCT) that assessed change in emotion processing and brain plasticity in healthy populations generally suffered from small sample sizes (range from 10 to 32 participants in the "active" condition) (Allen et al., 2012; Desbordes et al., 2012; Farb et al., 2010; Kral et al., 2018; Leung et al., 2018; Weng et al., 2013; Weng et al., 2018), which compromises the generalizability of the findings (Fox et al., 2016; Fox et al., 2014; Guendelman et al., 2017; Young et al., 2018). In addition, most studies focused on interventions that integrate a range of different contemplative practices and largely lacked the direct comparison with other meditation-based control conditions (Tang et al., 2015). It thus also remains unclear whether different types of mental practice, pursuing different aims, can induce selective changes in emotional reactivity (and implicit emotion regulation) at both neurofunctional and behavioural levels. 
To close this gap, we enrolled 332 participants in the context of the ReSource Project (Singer et al., 2016), a 9-month longitudinal mental training study. Based on the considerations made above, the ReSource project differentiated between three distinct 3-month training modules designed to cultivate (1) present-moment focused attention and interoceptive awareness (Presence module); (2) socio-affective skills, such as compassion, gratitude, prosocial motivation, and dealing with difficult emotions (Affect module) and (3) sociocognitive skills, such as metacognition and perspective-taking on self and others (Perspective module) (Figure 1a). The participants were assigned to one of the three training cohorts (TCs) who underwent the different modules in counterbalanced order or to the retest control cohort (RCC) (Figure 1b). This design allows for the comparison of differential effect of the training modules, with the training modules acting as "active" control groups for each other, and also against retest controls. We examined training-related changes of subjective and neural emotion processing when participants watched positive, negative and neutral stimuli using an emotion anticipation task (EmoAnt) (Somerville et al., 2012). In the EmoAnt, participants viewed emotional pictures that could be presented in predictable (i.e., they were preceded by a countdown) or unpredictable (i.e., preceded by a random countdown) manner, with no regulation instruction to further explore the impact of the different trainings on pre-emptive emotion control. Notably, we used emotional pictures with social content only (e.g., human suffering for negative pictures) rather than more "basic" emotions such as fear of snakes or spiders, as the modules Affect and Perspective heavily focus on training socio-affective and socio-cognitive capacities. After each block of pictures, the participants had to rate their emotions (from negative to positive) as well as their nervousness (from extremely nervous to not nervous at all) (Figure 1c). We used these assessments to measure the two core dimensions of emotions: valence and arousal (Baucom et al., 2012; Feldman Barrett and Russell, 1998; Lindquist et al., 2012). 
Based on previous literature using similar tasks, we predicted that at baseline (i.e., before the mental training), participants would exhibit increased amygdala activity for emotional vs. neutral pictures (Ochsner et al., 2002; Phan et al., 2002; Phelps and LeDoux, 2005; Somerville et al., 2012). Processing of positive emotion would be also accompanied by increased activation of VS, NAcc and OFC areas, while processing of negative emotion would be associated with activation of the amygdala and lateral regions of the PFC (Kragel and LaBar, 2016; Lindquist et al., 2012; Phan et al., 2002; Preckel et al., 2019). We expected module specific effects on behaviour and brain function: Similar to previous MBI studies (Allen et al., 2012; Desbordes et al., 2012; Kral et al., 2018), the effect of the Presence module should be associated with an improvement of top-down emotional control, manifesting in decreased negative affective ratings and a modulation of the activation of prefronto-limbic regions; The effect of the Affect module should be similar to the one observed after training of lovingkindness or compassion meditation (Desbordes et al., 2012; Engen and Singer, 2015; Klimecki et al., 2014), i.e. increased positive ratings and increased activation in brain areas associated with positive affect and affiliation, such as the ventral OFC, the VS and midbrain. Finally, we did not have specific a-priori hypotheses regarding the training of socio-cognitive skills (i.e., Perspective module) on emotion processing due to the lack of previous intervention studies in that domain. In addition to specific effects of different modules, we explored the overall 9months training effect, speculating in the existence of a cumulative effect of each module leading to less negative affect along with an improvement of the prefrontal control over limbic regions, as well as enhanced positive affect and increased activity in the VS, OFC and midbrain regions. 

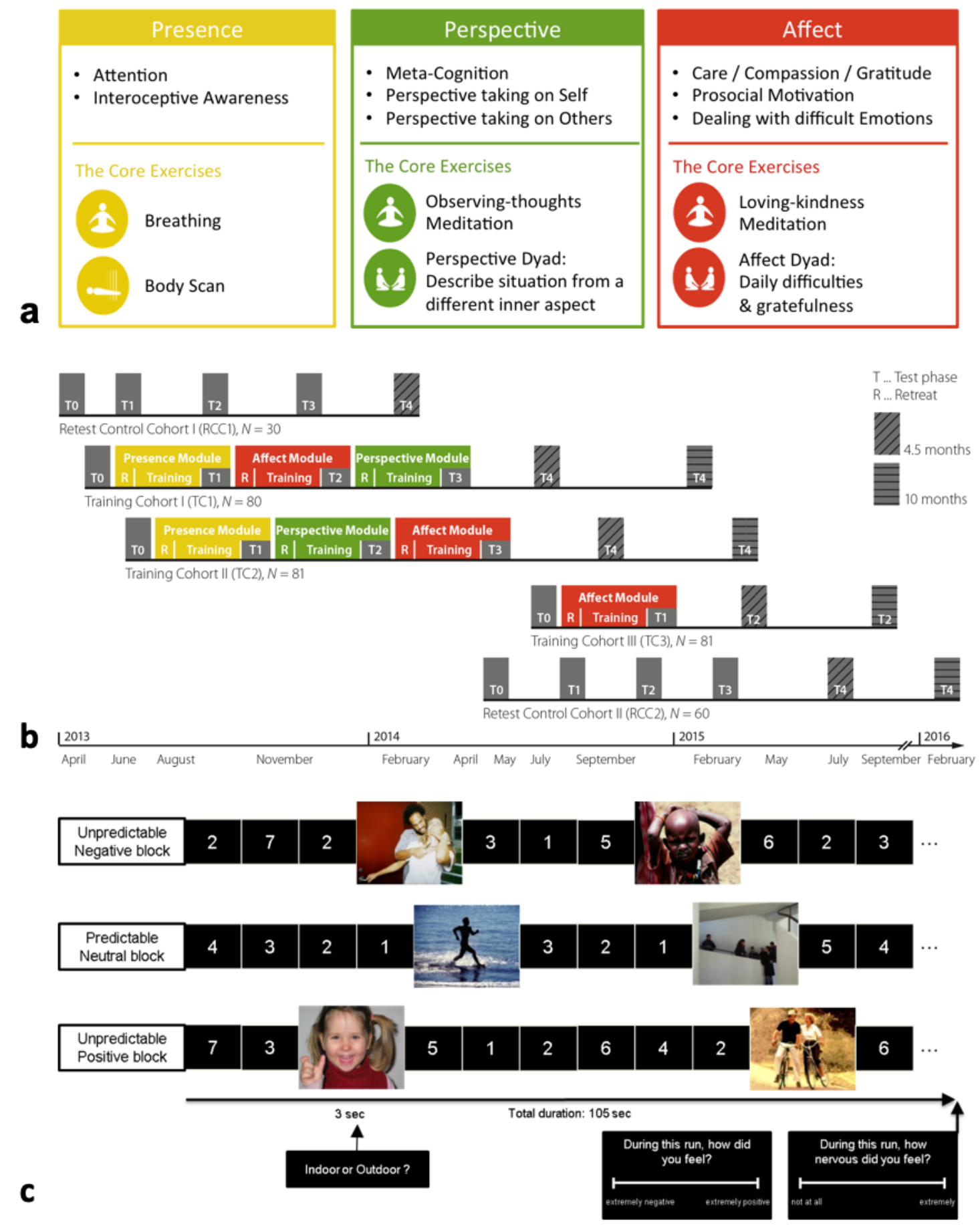

Figure 1: Design of the study. (a) Timeline of training (coloured areas) and data collection (grey areas) for the training and retest control cohorts. After baseline testing (T0), participants completed the modules in different orders. Training cohort 1 and 2 trained Presence first and then Affect and Perspective in a switched manner. Training cohort 3 trained Affect only. Retest cohorts completed the measurements without any training. They were tested in two cohorts but are analysed jointly. The full ReSource Design as shown in the figure also included follow-up assessments, but these are not included in the present study. (b) Illustration of the three 
modules of the program and their core exercises: Presence (yellow), Affect (red), Perspective (green). (c) Illustration of the experimental design. In each block negative, neutral or positive pictures were presented during three seconds. The apparition of the pictures was either predictable (ordered countdown) or unpredictable (random countdown). After each block, participants rated their own affect and nervousness. Panels a) and b) were adapted from Singer et al., (2016).

\section{Methods}

\subsection{Participants}

A total of 332 healthy participants (197 females; mean age $=40.74, \mathrm{SD}=9.24$; age range $=20-55)$ were recruited in the ReSource project. They were selected and assigned to one of the three training cohorts (TCs) or to the retest control cohort $(\mathrm{RCC}(\mathrm{N}=90), \mathrm{TC} 1(\mathrm{~N}=80)$, TC2 $(\mathrm{N}=81)$ or TC3 $(\mathrm{N}=81))$ using a bootstrapping process which ensured that all cohorts were matched for age, gender, marital status, income, IQ, and a number of self-reported personality traits(Singer et al., 2016). The final sample size per cohort, time point and measure vary due to study dropout/exclusion, partial dropout/exclusion from MRI experiments and technical, health, or scheduling issues at individual assessments. Finally, since the behavioural analysis focused on change scores (see below), the sample of the analysis was restricted to participants and time intervals where both pre- and post-scores were available, which leads to a final sample of 285 subjects ranged between 59 to 76 participants per group and time point (see Table 1 and Table S1 in Supplemental Material for dropout details).

All participants gave informed consent prior to participation. The study was approved by the Research Ethics Committee of the University of Leipzig (number 376/12-ff) and the Research Ethics Committee of Humboldt University of Berlin (numbers 2013-02, 2013-29, and 2014-10). The study was registered with the Protocol Registration System of ClinicalTrials.gov under the title "Plasticity of the Compassionate Brain" with the ClinicalTrials.gov Identifier: NCT01833104. 
Table 1. Sample description for available change scores

\begin{tabular}{|c|c|c|c|c|c|c|c|c|c|c|}
\hline & \multicolumn{4}{|c|}{ T0 to T1 } & \multicolumn{3}{|c|}{ T1 to $T 2$} & \multicolumn{3}{|c|}{$\mathrm{T} 2$ to $\mathrm{T} 3$} \\
\hline & RCC & TC1 & TC2 & TC3 & RCC & TC1 & TC2 & RCC & TC1 & TC2 \\
\hline $\mathrm{N}$ & 76 & 70 & 70 & 69 & 68 & 64 & 66 & 67 & 59 & 67 \\
\hline \multirow[t]{2}{*}{ Age } & 39.97 & 40.91 & 41.16 & 39.85 & 39.40 & 40.36 & 40.88 & 39.85 & 40.30 & 40.43 \\
\hline & \pm 9.21 & \pm 9.00 & \pm 9.90 & \pm 9.15 & \pm 9.32 & \pm 9.05 & \pm 10.10 & \pm 9.19 & \pm 9.39 & \pm 10.18 \\
\hline$\%$ Female & 55.26 & 57.14 & 60.00 & 56.52 & 52.94 & 51.56 & 59.09 & 52.24 & 47.46 & 58.21 \\
\hline
\end{tabular}

Notes: $\mathrm{RCC}=$ Retest Control Cohort, $\mathrm{TC} 1=$ Training Cohort $1, \mathrm{TC} 2=$ Training Cohort $2, \mathrm{TC} 3=$ Training

Cohort 3.

\subsection{Study design}

Four cohorts were set up for the ReSource project. The first two training cohorts (TC1 and TC2) underwent 9-month contemplative training with three 3-month modules practiced in different order (see description below). They both started with the Presence module. Subsequently, TC1 completed the Affect module followed by the Perspective module and TC2 did the Perspective module followed by the Affect module. The third training cohort (TC3) only trained a 3-month Affect module. The retest control cohort (RCC) did not carry out any mental training but was tested at the same time intervals as the TCs (i.e., four times at three months intervals) (Figure 1b).

\subsubsection{Trainings}

Each of the three modules of the ReSource program lasts 3 months, begins with a 3-day intensive retreat, includes 13 weekly group sessions of $2 \mathrm{~h}$ accompanied by experienced teachers, and about 30 min of daily practice. Each module has two core exercises, which participants were asked to practice five times a week.

Briefly, the Presence module aims at training attention, present moment awareness and interoceptive awareness. The two core exercises are "breathing meditation" and "body scan". In the breathing meditation, participants focus their attention on their breathing and in the body 
scan, participants focus on body sensations. The Affect module aims at cultivating an attitude of kindness and compassion toward oneself and others as well as to approach difficult emotions with acceptance, benevolence and gratitude. The core exercises are "loving-kindness meditation" and "Affect Dyads". The loving-kindness meditation aims at developing the attitude of care and compassion. In the Affect dyads, participants share difficult or grateful situation with other participants, taking one after the other the role of the listener and the speaker, thus promoting socio-emotional capacities. The purpose of the Perspective module is to train metacognition and cognitive perspective taking on the self and others. The core exercises are "observing-thoughts meditation" and "Perspective Dyads" in which participants practice inner perspective taking (i.e., re-experiencing a recent situation from a different perspective) as well as perspective taking on others (i.e., taking the perspective of the dyadic partner). The Perspective module aims at training socio-cognitive abilities. Figure 1a illustrates the content of the three modules (see Singer et al., 2016 for a detailed description of the content of each module).

\subsubsection{Stimuli and task}

We used a modified version of the EmoAnt task developed by Somerville et al. (2012). In the original version, Somerville et al. (2012) used negative and neutral stimuli depicted both social and non-social scenes from the International Affective Picture System (IAPS) database(Lang et al., 1999). Here, we selected positive, negative and neutral stimuli with social scenes only. They were extracted from the IAPS, the Geneva Affective Picture Database (GAPED) (Dan-Glauser and Scherer, 2011) and the Emotional Picture Set (EmoPicS) (Wessa et al., 2010). Different sets of stimuli were built for each time point. Each set contained 28 positive pictures, 28 negative pictures and 28 neutral pictures with comparable valence and arousal, as defined by the norms of the databases (see supplemental material, Table S2). In each set, half of the pictures were taking place indoors and the other half outdoors. 
While undergoing fMRI scanning, participants were presented with these stimuli. To ensure focused processing of the stimuli, participants judged whether each picture depicted an indoor or outdoor scene in a total of six blocks of 14 stimuli each. These blocks varied in the valence of the presented stimuli (positive, negative, neutral) and also in the predictability of stimulus presentation. To implement this, each stimulus was preceded by a 2-8 second "countdown" in which numbers were presented (length of countdown was assigned pseudorandomly). In the "predictable" condition, these numbers accurately represented the number of seconds remaining before picture onset. In the "unpredictable" condition, numbers were randomly presented, providing no predictive information regarding picture onset (Figure 1c). This resulted in six experimental conditions: ordered-positive, ordered-negative, orderedneutral, random-positive, random-negative and random-neutral. Condition sequence was random for each participant. Half of the stimuli per valence were assigned randomly to the predictable or non-predictable condition.

Before fMRI acquisition, participants were trained to perform the task with different stimuli than those used during the experiment. Each block began with a $5 \mathrm{~s}$ start cue (fixation cross) followed by $3 \mathrm{~s}$ instructions informing participants of the forthcoming block type. Following the instructions, stimulus presentation continuously alternated between countdowns (1 s per number; jittered from 2 to 8 numbers) and picture presentation $(3 \mathrm{~s})$. Participants responded by using an MRI-compatible response box, using the index and middle finger on the right hand. Each block ended with a 3 s stop cue (fixation cross) followed by a rating period ( 5 s) in which participants used a continuous scale from none to very much (coded from 0 to 500) indicating 1$)$ how negative to positive they felt $(0=$ extremely negative, $250=$ neutral, $500=$ extremely positive $)$ and 2$)$ how nervous they felt during the block $(0=$ not at all nervous, 500 $=$ extremely nervous). The total duration of the experiment was $13 \min 30 \mathrm{~s}$. 


\subsection{Analysis of behavioural measures}

The subjective emotional valence and nervousness ratings were considered for behavioural analyses. Data were analysed using R software (R Core Team, 2015). Following our hypotheses three separate linear mixed models (LMM) (lme4 package (Bates et al., 2015)) analyses were performed for both measures:

Data at baseline (i.e., T0) were entered in an LMM including fixed effects for the Valence (positive, negative, neutral) and Predictability (ordered countdown, random countdown) of the stimuli and random intercepts for participants:

T0 Rating $=\beta 0+\beta 1 *$ valence $+\beta 2 *$ predictability $+\beta 3 *($ valence by predictability $)+$ random

$$
\text { effect (participant) }
$$

In order to assess the specific effect of the modules on emotion processing in the same way as in the fMRI analyses (see below), we computed two new dependent variables for each participant by subtracting average ratings for neutral pictures from those for negative and positive pictures (i.e., [negative - neutral] and [positive - neutral]). Then, for each participant and each module, we calculated a change score by subtracting individual scores before the module from the scores at the end of the module. These change scores were entered into an LMM including fixed effects for each module at a given time interval and random intercepts for participants:

$$
\begin{gathered}
\text { Change score }=\beta 0+\beta 1 * \text { Re-test } 2+\beta 2 * \text { Re-test } 3+\beta 3 * \text { Presence }+\beta 4 * \text { Affect } 1+\beta 5 * \text { Affect } 2 \\
+\beta 6^{*} \text { Affect } 3+\beta 7 * \text { Perspective } 2+\beta 8 * \text { Perspective } 3+\text { random effect (participant) }
\end{gathered}
$$

The first retest interval (i.e., Re-test1) is the intercept of the model, so all other effects are estimated in relation to this baseline. The models allow to test the effects of the trainings by contrasting the respective parameter estimates against each other. Each module was contrasted against effects of other modules and of retest (i.e., Presence vs. Re-test (T0 to T1), Presence vs. Affect (T0 to T1), Affect vs. Re-test (all time points), Perspective vs. Re-test and Affect vs. 
Perspective (T1 to T3), see Table S3 in the supplemental material for the description of the contrast matrix.

Similarly, to test for the overall effect of the 9-month program, data before and after the training were entered into an LMM including fixed effects for the Training (TC1 and TC2 vs. RCC) and the Timepoint (T0 vs. T3) as well as random intercepts for participants. As above, dependent variables consisted of subjective emotional valence and nervousness ratings for positive and negative as compared to neutral pictures:

Ratings $=\beta 0+\beta 1 *$ training $+\beta 2 *$ timepoint $+\beta 3 *($ training by timepoint $)+$ random effect

\section{(participant)}

As an estimate of effect size, for each analysis and each contrast, we provide the models estimates $(b)$.

\subsection{Analysis of fMRI measures}

\subsubsection{MRI acquisition}

MR images were acquired on a whole-body 3T Siemens Verio Scanner (Siemens Medical Systems, Erlangen, Germany) using a 32-channel head-coil. Functional images were acquired with gradient-echo/T2* weighted EPI sequence $(\mathrm{TR}=2000 \mathrm{~ms}, \mathrm{TE}=27 \mathrm{~ms}$; flip angle $=$ $77^{\circ} ; 37$ axial slices with $1 \mathrm{~mm}$ gap tilted $\sim 30^{\circ}$ from the bi-commissural plane; FOV $=210 \mathrm{~mm}$; matrix size $=70 \times 70$; in plane voxel size $=3 \times 3 \mathrm{~mm}$; slice thickness $=3 \mathrm{~mm}$; 405 volumes) High resolution structural images were acquired with a T1-weighted 3D-MPRAGE sequence $\left(\mathrm{TR}=2300 \mathrm{~ms}, \mathrm{TE}=2.98 \mathrm{~ms}, \mathrm{TI}=900 \mathrm{~ms}\right.$, flip angle $=9^{\circ} ; 176$ sagittal slices, $\mathrm{FOV}=256 \mathrm{~mm}$, matrix size $=240 \times 256$, slice thickness $=1 \mathrm{~mm}$; total acquisition time $=5.10 \mathrm{~min})$. We also acquired B0 field maps using a double-echo gradient-recalled sequence with matching dimensions to the EPI images $(\mathrm{TR}=488 \mathrm{~ms}, \mathrm{TE}=4.49$ and $6.95 \mathrm{~ms})$. 


\subsection{2. fMRI preprocessing}

Preprocessing steps were performed by using the SPM12 software package (Welcome Department of Imaging Neuroscience, Institute of Neurology, London, UK), running on Matlab 8.6 (R2015b) (Mathworks, Natick, MA, USA). Functional images were first realigned (using rigid body transformations) and unwarp to additionally correct for distortion using B0 field maps and were subsequently time-corrected (slice timing). Then, the T1-weighted anatomical volume was coregistered to mean image created by the realignment procedure and was segmented. Finally, the functional images were normalized to the MNI space using DARTEL procedures (Ashburner, 2007) and smoothed using 8-mm full-Width at half maximum Gaussian.

\subsubsection{First level fMRI analyses}

For each participant, experimental conditions (ordered positive, ordered negative, ordered neutral, random positive, random negative, random neutral) were modelled using the General Linear Model (GLM). Movement-related parameters and outliers identified using the ART toolbox (http://nitrc.org/projects/artifact_detect/) were also included as covariate of no interest. The blood-oxygen-level dependent response for each event for the six different conditions was modelled using a canonical form of the hemodynamic response function (HRF) together with the time and dispersion derivatives. Before estimation, a high-pass filtering with a cut-off period of $128 \mathrm{~s}$ was applied.

\subsubsection{Second level fMRI analyses}

We performed three separated analyses on the second level to identify: (1) the cerebral networks involved in the task before the training (i.e., at T0); (2) the specific brain plasticity induced by each module; and (3) neural modulation induced by the overall 9-month training.

1) We used a 3 x 2 repeated-measure factorial analysis using GLMFlex (http://mrtools.mgh.harvard.edu/) to test the effect of the experimental factors at baseline (i.e., 
T0). We evaluated the main effect of the Valence, the main effect of the Predictability and the interaction Valence-by-Predictability as well as Valence specific activations (i.e., [negative vs. neutral] and [positive vs. neutral]). The results were thresholded at $p<0.001$ (uncorrected) at the voxel level and corrected for multiple comparisons using cluster extent family-wise error rate (FWE) correction at $p<0.05$ as implemented in the SPM toolbox, which led to an extend threshold $\mathrm{k}>101$ voxels.

2) We used the Sandwich Estimator (SwE) method as implemented in the SwE toolbox (http://warwick.ac.uk/tnichols/SwE) (Guillaume et al., 2014) to assess the effects of each modules on brain activity with the following contrasts: Presence vs. Re-test, Presence vs. Affect, Affect vs. Re-test, Perspective vs. Re-test and Affect vs. Perspective (see Table S3 in supplemental material for the description of the contrast matrix). Since no significant effect of Predictability or Predictability-by-Valence interaction was observed at T0, the analyses focused on the modules effect for [negative vs. neutral] and [positive vs. neutral] first level contrasts. Contrast maps for each subject and time points were then entered in a single model. The fitted models allow to test the above specified hypotheses by contrasting the respective parameter estimates against each other. The results were thresholded at $p<0.001$ (uncorrected at the voxel level) and were corrected for multiple comparisons using wild bootstrap procedure (999 iterations) and cluster extent family-wise error rate (FWE) correction at $p<0.05$ as implemented in the SwE toolbox (Guillaume et al., 2014), which led to an extend threshold k $>98$ voxels.

Second-level functional activation maps were also overlaid with empathy and ToM networks identified in a previously published study on subsample data from the participants at baseline (i.e., the EmpaToM task) (Kanske et al., 2015). We used the EmpaToM task contrast during negative vs. neutral socio-emotional video to obtain an "empathy mask" and contrast between ToM and non-ToM questions for "ToM mask". 
3) We tested for the overall effect of the 9-month program with a $2 \times 2$ factorial design, including the Cohort (TC1 and TC2 vs. RCC) and the timepoint (T0 vs. T3) modelled with the SwE toolbox.

All second level analyses were constrained to voxels within a grey matter mask derived from the group DARTEL-generated template thresholded at 90\% grey matter probability. Brain regions involved in different contrasts were labelled by means of macroscopic parcellation of the MNI single subject reference brain (Tzourio-Mazoyer et al., 2002)

\section{Results}

\subsection{Behavioural results}

\subsubsection{Behavioural results at baseline}

The analysis of subjective emotional valence ratings revealed a significant main effect of Valence $[F(2,5659.7)=5802.5, p<0.001]$. As expected, the negative stimuli were judged more negative than the neutral $(z=52.40, p<0.001)$, and the positive stimuli more positive than the neutral $(z=22.78, p<0.001)$. However, the analysis did not reveal a significant effect of Predictability $[F(1,5659.7)=1.40, p=0.24]$ or a Predictability-by-Valence interaction $[F(2$, $5659.7)=1.10, p=0.33]$

Regarding nervousness ratings, we also observed a significant main effect of Valence $[F(2,5668.4)=1020.82, p<0.001]$. Participants reported to be more nervous for negative vs. neutral stimuli $(\mathrm{z}=-26.34, p<0.001)$ as well as for neutral vs. positive stimuli $(\mathrm{z}=-3.60$, $p<0.001)$. Similarly, we did not observe a significant effect of Predictability $[F(1,5668.4)=$ $0.77, p=0.38]$ or a Predictability-by-Valence interaction $[F(2,5668.4)=0.76, p=0.47]$.

\subsubsection{Behavioural change induced by the training modules}

Since we did not observe significant effects of Predictability or Predictability-byValence interaction at $\mathrm{T} 0$, analyses of modules training effects focused only on the effect of the 
Valence of the stimuli. To test for the specific effects of the modules, for each participant and each module, we calculated a change score by subtracting individual scores before the module from the scores at the end of the module.

Regarding subjective emotional valence ratings for [negative - neutral], from T0 to T1, effects of Presence was not different from retest $(b=-15.73, z=-1.39, p=0.16)$ and Affect $(b$ $=12.32, z=1.06, p=0.29$ ), however, ratings were significantly more negative after retest than after Affect ( $b=28.05, z=2.13, p=0.03)$, suggesting that the Affect training "buffered" the increase of subjectively experienced negative affect after repeating the task a second time. Subsequent comparisons revealed an overall significant decrease in negative ratings after $A f f e c t$ from T0 to T3, which was significantly different from retest $(b=-55.13, z=2.13, p=0.02)$ (Figure 2, Panels a and b). The model for subjective emotional valence ratings for [positive neutral] revealed significantly increased positive ratings after Perspective in comparison to retest $(b=37.29, z=2.17, p=0.03)$.

The model for the change in nervousness rating for [negative - neutral] revealed increase nervousness from T1 to T3 after Perspective in comparison to both retest $(b=-72.56$, $z=-2.63, p=0.009)$ and Affect $(b=78.98, z=2.83, p=0.005)$ (Figure S1, Panels a and $b)$. There was no significant differential effect of the modules on nervousness rating for [positive - neutral] ratings.

Distributions of behavioural changes after the training modules are presented in Figure S2, descriptive statistics are reported in Table S4 and detailed statistics for each contrast and measure are reported in Table S5. 

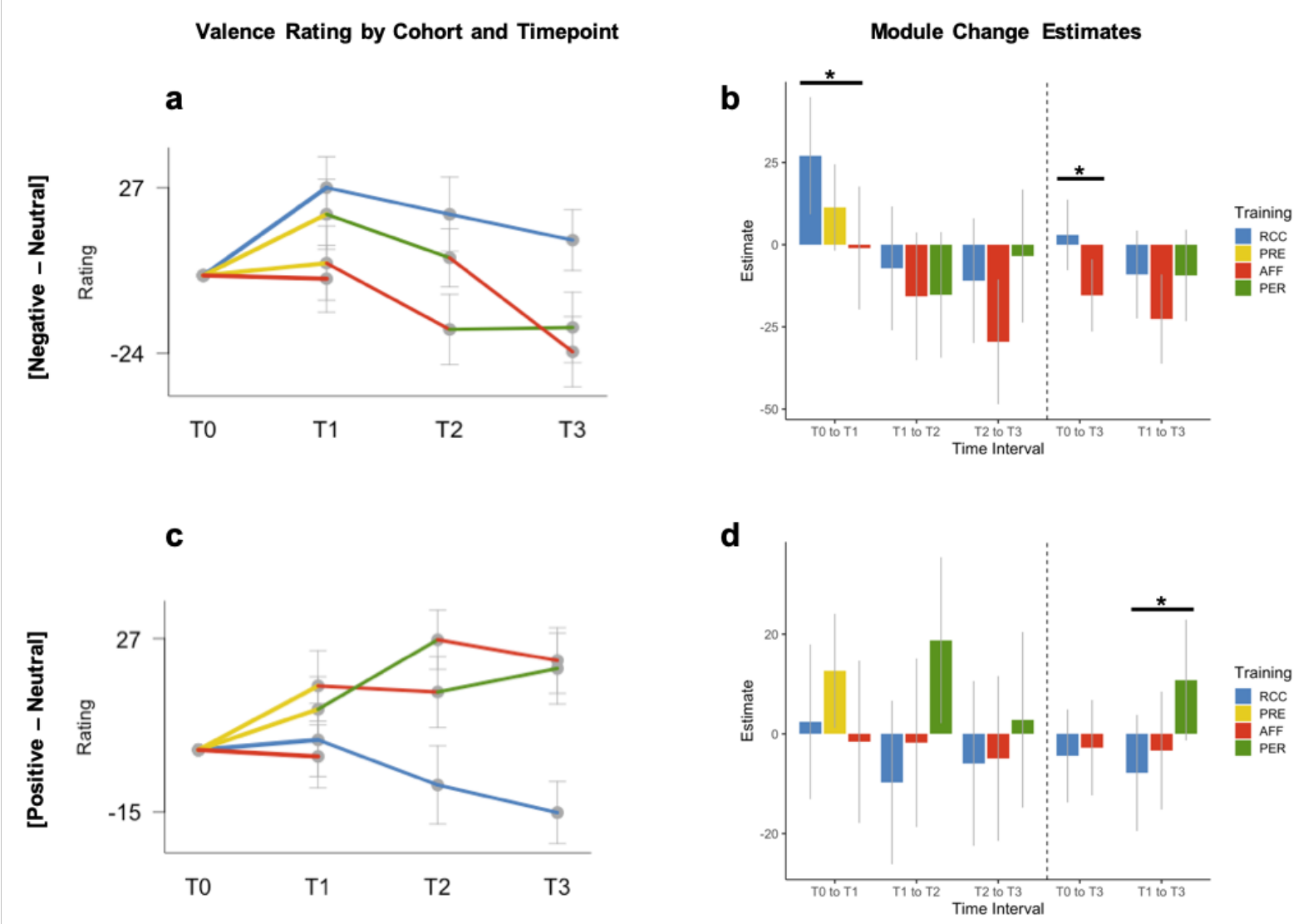

Figure 2: Descriptive plots of the change in valence rating in the course of the 9-month training. (a-b)

Change in [negative - neutral] ratings. (c-d) Change in [positive - neutral] ratings. The line-graphs show ratings according to the group and time point. Values at the first measurement point are equalized representing statistical control for baseline scores. The histograms depict the estimated effect of each module $(\mathrm{RCC}=$ retest, $\mathrm{PRE}=$ Presence, $\mathrm{AFF}=$ Affect, $\mathrm{PER}=$ Perspective $)$ To the right of the dashed lines, estimates are averaged across time intervals as it was done to test the overall effect of the modules across time points. Error bars represent $95 \%$ confidence intervals. ${ }^{*} p<0.05$.

$N B$ : as subjective valence ratings range from negative to positive $(0=$ extremely negative, $250=$ neutral, $500=$ extremely positive), for illustrative purposes, panels a and b depicted actually the difference between neutral minus negative scores.

\subsubsection{Behavioural change after the overall 9-month training}

We found a significant interaction between Training and Timepoint for both [negative - neutral] $[F(1,213.26)=4.17 ; p=0.04]$ and [positive - neutral] subjective valence ratings $[F(1$, $211.85)=16.91 ; p<0.0001$. Planned comparisons revealed that the ratings for [negative - 
neutral] were significantly less negative from T0 to T3 for the TCs $(b=-18.53, z=-2.57, p=$ $0.01)$, whereas there was no change for the RCC $(b=6.43, z=0.65, p=0.51)$ (Figure S3, Panel a). In addition, ratings for [positive - neutral] were significantly more positive from T0 to T3 for the TCs $(b=22.37, z=4.02, p<0.0001)$ and significantly less positive for the RCC $(b=-$ $16.53, z=-2.16, p=0.03$ ) from T0 to T3 (Figure S3, Panel b).

Regarding nervousness ratings, the interaction training-by-timepoint was only marginally significant for [negative - neutral] ratings $[F(1,215.57)=3.88 ; p=0.05]$ and not significant for $[$ positive - neutral] ratings $[F(1,435)=1.22 ; p=0.23]$ (Figure S3, Panels c and d).

\section{2. fMRI results}

\subsection{1. fMRI results at baseline}

In comparison to neutral stimuli, negative stimuli yielded increased activation in the ventral visual pathway; i.e., in two bilateral clusters encompassing the inferior occipital, temporal and parietal gyri, as well as in the bilateral supramarginal gyrus (SMG), the right inferior frontal gyrus (IFG) and in a large cluster including left amygdala, insula and superior temporal gyrus $(\mathrm{STG})\left(p_{\text {unc(voxel) }}<0.001, p_{\mathrm{FWE}(\text { cluster })}<0.05\right)$. For exploratory purposes, we also looked at the results with an uncorrected threshold $\left(p_{\text {unc(voxel })}<0.001, \mathrm{k}>10\right)$. We observed increased activation in the superior and middle prefrontal cortex as well as in the orbitofrontal cortex, the middle insula, the middle and posterior cingulate cortex and in the right amygdala at this lower threshold (Figure 3a, Table 2). Positive stimuli induced increased activation of the ventral visual pathway and in the posterior cingulate cortex/precuneus $\left(p_{\text {unc(voxel })}<0.001\right.$, $\left.p_{\text {FWE(cluster) }}<0.05\right)$. Exploratory results show that the left angular gyrus, the right STS and bilateral IFG were also marginally more strongly activated in response to positive vs. neutral stimuli $\left(p_{\text {unc(voxel) }}<0.001, \mathrm{k}>10\right.$; Figure $3 \mathrm{~b}$, Table 2$)$. We did not observe a modulation of the brain activity depending on the predictability of the stimuli and no interaction of Valence by Predictability. 
Table 2. Activation peaks before the training (T0)

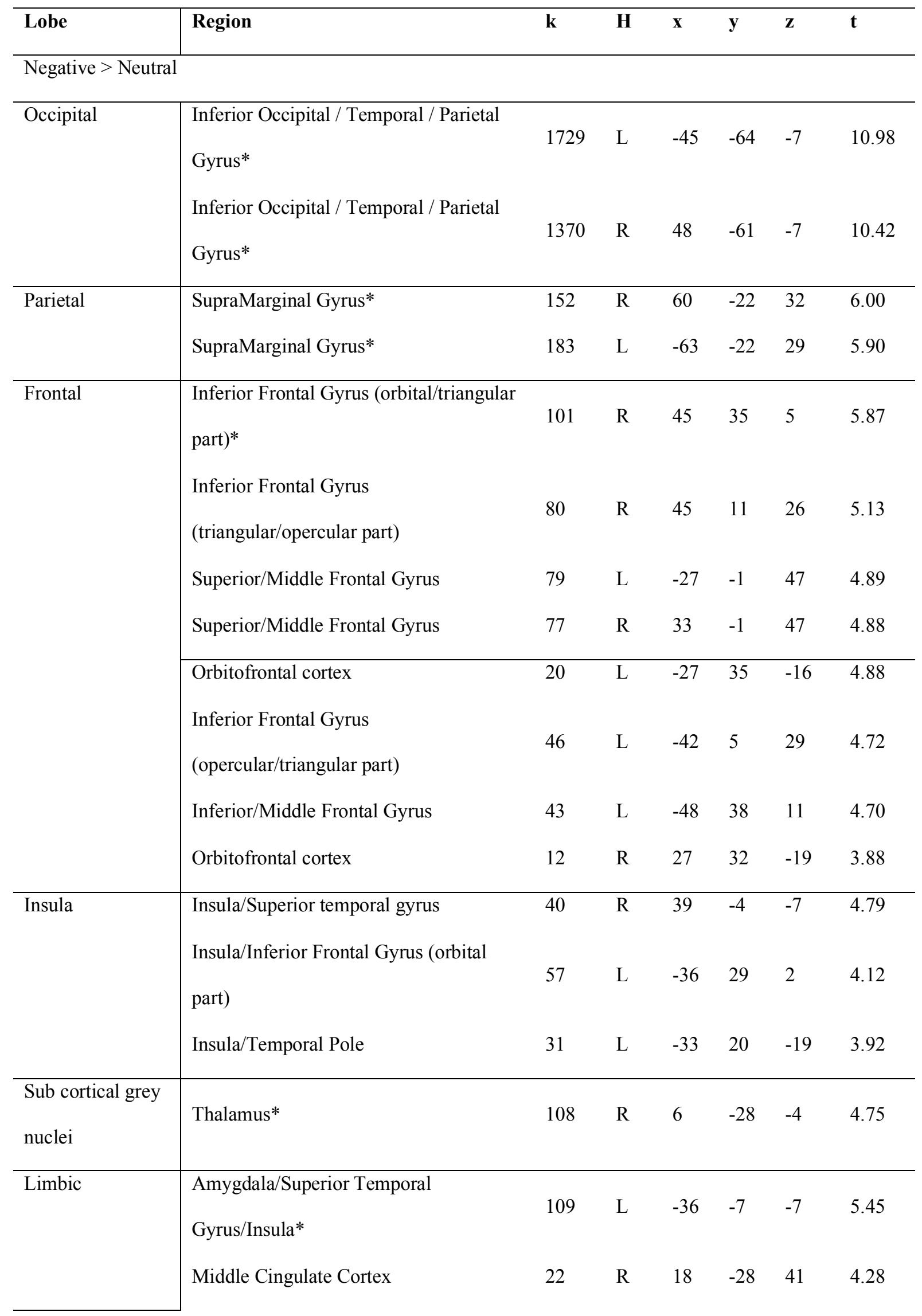




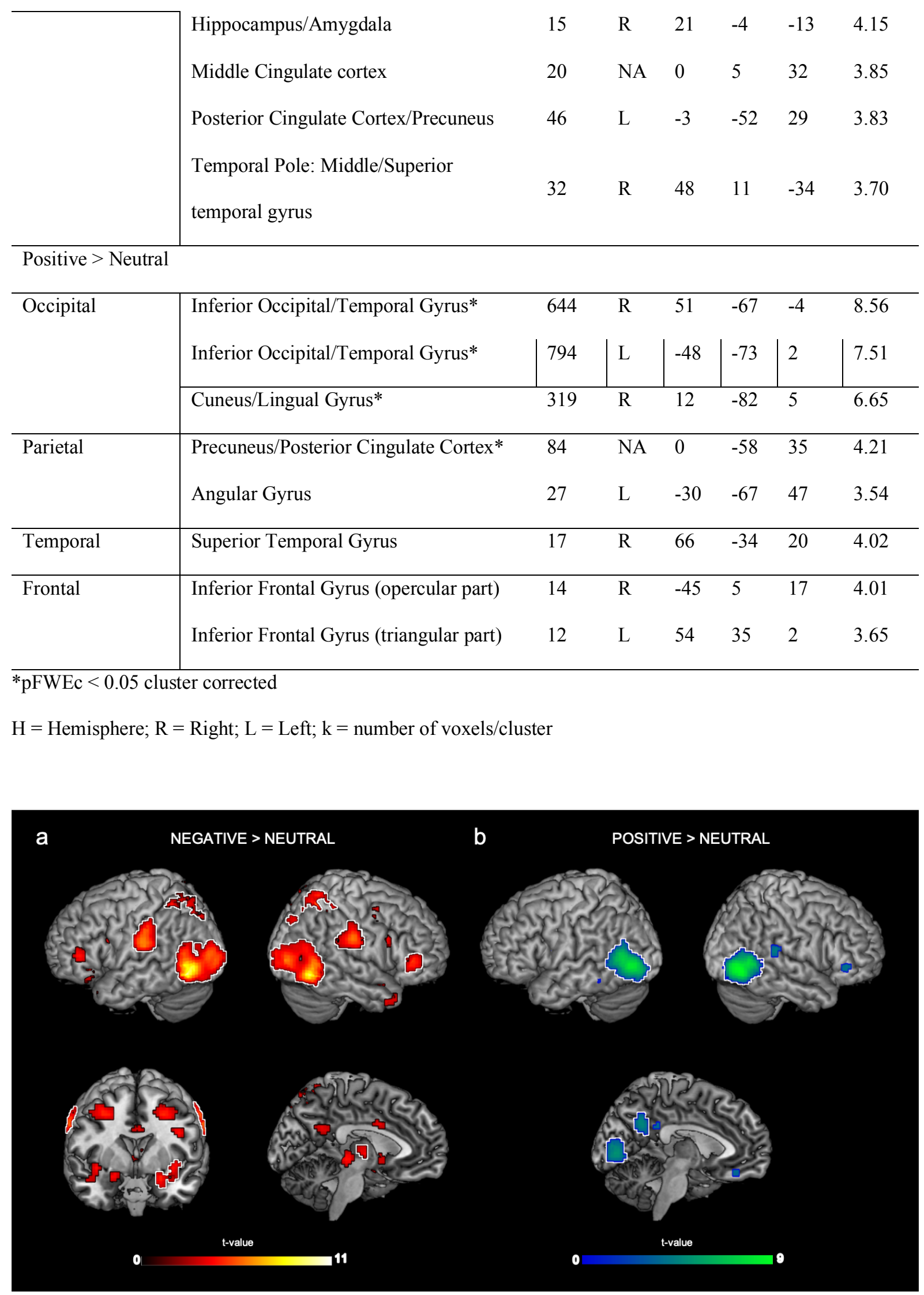

Figure 3: Cerebral responses to emotional scenes at baseline (T0). Brain activation resulting from the contrast [negative $>$ neutral] are represented in red-yellow scale (a) and those of the contrast [positive $>$ neutral] 
in blue-green scale (b). Findings are thresholded at $p_{\text {uncor }}<0.001, k>10$ and clusters that survived correction for multiple comparison $\left(p_{\mathrm{FWEc}}<0.05\right)$ are surrounded with a white line.

\subsubsection{Neurofunctional change induced by the training modules}

In comparison to Retest, participants who trained Affect showed significantly increased activation in the right SMG when processing negative vs. neutral stimuli $\left(p_{\text {unc(voxel })}<0.001\right.$, $\left.p_{\text {FWE(cluster) }}<0.05\right)$. To test for wider network effects, we also investigated non-corrected trends on $p_{\text {unc(voxel) }}<0.001$ levels, for illustrative purpose only. This revealed that participants also tended to show increased activity in several lateral prefrontal regions, comprising two clusters in the right IFG, one cluster in the left IFG, two clusters in the right middle frontal gyrus and additionally in three clusters situated in the right inferior temporal gyrus and in the bilateral occipital cortex (cuneus) $\left(p_{\text {unc(voxel })}<0.001, \mathrm{k}>10\right.$, Figure 4, Panel a) and b); Table 3). There was no significant change in brain activity after Affect training for positive vs. neutral stimuli. Training Perspective did not lead to significant neurofunctional modulation for either [negative vs. neutral] and [positive vs. neutral] contrasts. There was no significant difference between training Affect and Perspective and between Presence and Retest for both contrasts.

To better understand our results and see if they relates to change in empathy or ToM processing, activation maps resulting from these second-level analyses were overlaid with functional activation maps from a previously published study on subsample data from the participants at baseline that aimed at measuring the cerebral correlate of empathy and ToM (i.e., the EmpaToM task) (Kanske et al., 2015). Interestingly, brain regions showing increased activation after Affect training overlapped partially with regulation regions of the empathy network, i.e., right SMG and right lateral prefrontal regions as reported in Kanske et al. (2015) (Figure S4a).

Exploratory analyses at an uncorrected threshold of $p_{\text {unc(voxel) }}<0.001, \mathrm{k}>10$ revealed partial overlap between temporal regions showing increased activity after Perspective training 
vs. Retest for the processing of positive vs. neutral stimuli and the ToM network as identified by Kanske et al. (2015) (see Figure S4b).

Table 3. Activation peaks of the change after Affect training vs. Re-test

\begin{tabular}{|c|c|c|c|c|c|c|c|}
\hline Lobe & Region & $\mathbf{k}$ & $\mathbf{H}$ & $\mathbf{x}$ & $\mathbf{y}$ & $\mathbf{z}$ & $\mathbf{Z}$ \\
\hline \multicolumn{8}{|c|}{ Negative $>$ Neutral } \\
\hline Parietal & SupraMarginal Gyrus* & 140 & $\mathrm{R}$ & 45 & -40 & 41 & 4.20 \\
\hline Temporal & Inferior Temporal Gyrus & 94 & $\mathrm{R}$ & 60 & -43 & -13 & 3.86 \\
\hline \multirow[t]{2}{*}{ Occipital } & Lingual Gyrus & 21 & $\mathrm{~L}$ & -18 & -52 & -1 & 3.86 \\
\hline & Cuneus/Lingual Gyrus & 11 & $\mathrm{R}$ & 12 & -76 & 32 & 3.44 \\
\hline \multirow{5}{*}{ Frontal } & $\begin{array}{l}\text { Inferior Frontal Gyrus (triangular } \\
\text { part) }\end{array}$ & 44 & $\mathrm{R}$ & 60 & 23 & 11 & 3.76 \\
\hline & $\begin{array}{l}\text { Inferior Frontal Gyrus (triangular } \\
\text { part) }\end{array}$ & 49 & $\mathrm{~L}$ & -48 & 20 & 8 & 3.58 \\
\hline & $\begin{array}{l}\text { Inferior Frontal Gyrus (triangular } \\
\text { part) }\end{array}$ & 12 & $\mathrm{~L}$ & -48 & 44 & 2 & 3.53 \\
\hline & Middle Frontal Gyrus & 15 & $\mathrm{R}$ & 48 & 14 & 41 & 3.42 \\
\hline & Middle Frontal Gyrus & 13 & $\mathrm{R}$ & 45 & 47 & 11 & 3.42 \\
\hline
\end{tabular}

*pFWEc $<0.05$ cluster corrected

$\mathrm{H}=$ Hemisphere; $\mathrm{R}=$ Right; $\mathrm{L}=$ Left; $\mathrm{k}=$ number of voxels/cluster

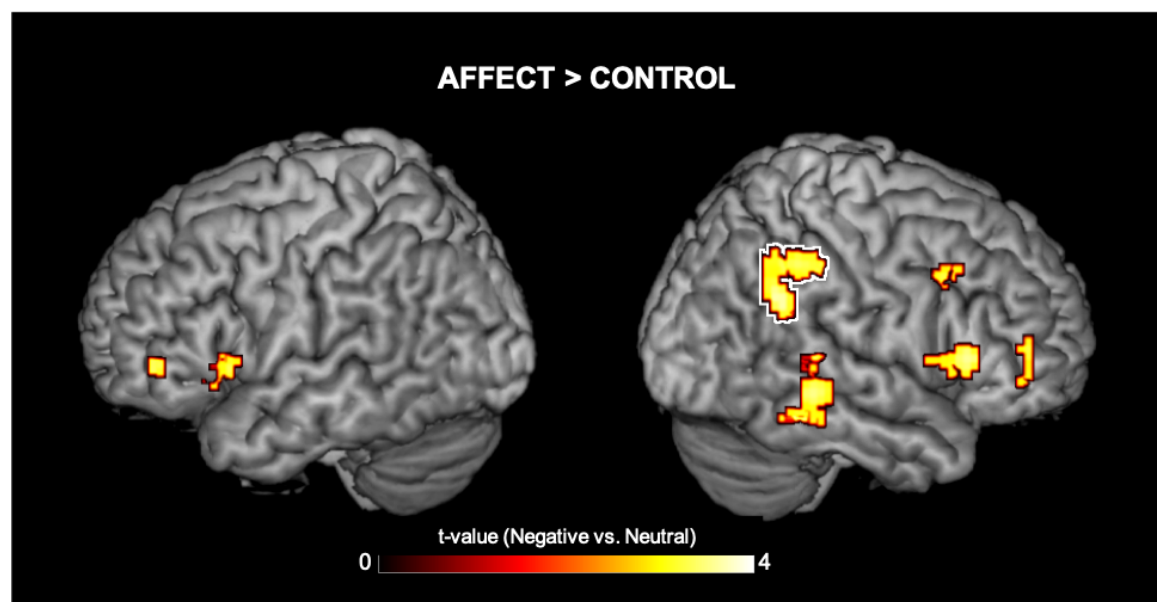

Figure 4: Modulation of the cerebral activity after Affect module training. Brain areas showing increase activity for the contrast [negative $>$ neutral] after the Affect module in comparison to re-test. Findings are thresholded at $p_{\text {uncor }}<0.001, \mathrm{k}>10$; cluster that survived $p_{\mathrm{FWEc}}<0.05$ correction are surrounded by a white circle. 


\subsubsection{Neurofunctional change after the overall 9-month training}

The contrast [positive $>$ neutral] revealed a significant Training-by-Timepoint interaction within a large cluster encompassing the left lingual gyrus and the posterior cingulate cortex $\left(p_{\text {unc(voxel) }}<0.001, p_{\mathrm{FWE}(\mathrm{cluster})}<0.05\right)$. For exploratory purposes, we investigated changes in brain activation after the training with non-corrected threshold $\left(p_{\text {unc(voxel) }}<0.001, \mathrm{k}>10\right)$. We additionally found increased activation within the bilateral superior temporal cortices (angular gyrus), the posterior cingulate cortex, the thalamus and the brainstem in the TCs vs. RCC (Figure S5, Table S6). The contrast [negative vs. neutral] did not show supra-threshold voxels for the interaction Training-by-Timepoint.

\section{Discussion}

We investigated the differential effects of three types of meditation-based mental training practices on emotional reactivity to positive and negative socio-emotional stimuli. In the context of the ReSource project(Singer et al., 2016), 332 participants took part in a 9-month long mental training study. They performed an emotion anticipation task(2012) while being scanned with fMRI before and after engaging in three 3-month modules focusing on improving (1) present-moment focused attention and interoceptive body awareness (Presence), (2) socioaffective skills, such as compassion, gratitude and coping with difficult emotions (Affect), or (3) socio-cognitive skills, such as perspective taking on self and others (Perspective). Before the training, the processing of both positive and negative vs. neutral socio-emotional stimuli elicited activation of the ventral occipito-temporal visual areas. Additional activation for negative vs. neutral stimuli was observed in lateral fronto-parietal and limbic regions, the amygdala and the insular cortex in particular. Notably, we showed that the mental training modules had differential effects on behavioural affect ratings after exposure to positive, neutral or negative pictures, as well as on underlying functional brain activation patterns. The Affect module led to decreased negative ratings when participants were exposed to negative pictures 
compared to neutral ones and increased activity in the right SMG. Surprisingly, we did not find specific behavioural or neurofunctional changes after Presence. We also found that after the 9month training, participants of the active TCs judged their affect more positively and less negatively compared to the participants of the RCC. They also revealed increased activation from baseline until the end of the 9-month training within midbrain and occipital areas when watching positive vs. neutral stimuli.

Before the training, neural activity during the task involved two different networks for positive vs. neutral and negative vs. neutral stimuli. In both emotional conditions, the ventral visual stream, mainly composed of lateral occipital and temporal cortex, as well as the amygdala, were activated. This suggests an enhancement of activation in the visual cortex when processing emotional stimuli, as ventral visual cortex responses in emotional contexts might be reinforced through feedback connections from the amygdala (Lang et al., 1998; Phan et al., 2002; Vuilleumier, 2005; Vuilleumier and Pourtois, 2007). In addition, the cerebral response to negative vs. neutral stimuli involved activation of lateral prefrontal (IFG/AI), limbic (amygdala, subcortical nuclei) and parietal regions (SMG). This pattern of results is coherent with what has been observed in other studies on processing of negative emotion (Kragel and LaBar, 2016; Lindquist et al., 2012; Phan et al., 2002; Preckel et al., 2019) or empathy (Bzdok et al., 2012; Kanske et al., 2015; Lamm et al., 2011; Singer et al., 2004). The processing of positive vs. neutral stimuli further involved midline cortical structures (ventro-medial PFC, PCC), a set of brain areas that are known to be involved in self-referential processes. We speculate that this could reflect the representation of prior experiences when faced with positive emotions of others (Buckner et al., 2008; Engen et al., 2017; Lindquist et al., 2011).

The result from the manipulation of unpredictable (i.e., random countdown) vs. predictable (i.e., ordered countdown) conditions was not conclusive, both at behavioural and neural levels. Unlike Somerville et al. (2012), we did not find a modulation of the cerebral 
activity depending on the predictability of the stimuli. This could be explained by an extensive screening for anxiety, mood symptoms and personality disorders when recruiting and selecting participants for the ReSource project (Singer et al., 2016). Indeed, in Somerville et al. (2012) the participants covered a wide range of anxiety scores, which was also correlated with their fMRI results, especially in the unpredictable condition.

Regarding our predictions for the specific effects of the Presence, Affect and Perspective training modules, unlike previous studies on MBI (Fox et al., 2016; Fox et al., 2014; Tang et al., 2015; Young et al., 2018) we did not find a training-related modulation of brain activity after the Presence training. In previous studies, the authors claimed that present-moment focused attention helps people to better regulate their emotions via a reinforcement of the topdown cognitive control (Allen et al., 2012; Farb et al., 2012; Kral et al., 2018; Lutz et al., 2014). In our study, the training of present-moment and attention-based meditation techniques did not lead to downregulation of negative emotion either at the behavioural nor at the cerebral level. It should be noted that the Presence module was not exactly equivalent to the classical MBI programs (e.g., MBCT, MBSR) tested in previous RCT (Allen et al., 2012; Desbordes et al., 2012; Farb et al., 2010; Kral et al., 2018). Here, the Presence module focused only on attentionbased mindfulness meditation, such as the body-scan or the breathing meditation, but did not include mindfulness practices focusing on acceptance, loving kindness, mindfulness on emotions or observing thoughts as in classical 8-weeks MBI. As the ReSource project aimed at differentiating between different types of meditation-based practices, practices explicitly associated to emotional processing were included in the Affect module and practices cultivating meta-cognitive capacities like observing thoughts were included in the Perspective module. This could explain why, unlike previous studies on mindfulness, we did not observe a modulation of emotional processing after pure attention-based practices such as implemented in the Presence training. 
Importantly, our results revealed that, in contrast to the retest control group, after the overall 9-month training and especially after the Affect training, participants reported feeling less negative affect after having being exposed to negatively valenced social pictures. Moreover, between $\mathrm{T} 0$ and $\mathrm{T} 1$, negative ratings increased for the retest control participants (i.e., RCC) but not in the cohort who trained Affect only (i.e., TC3). Thus, for retest-control subjects, the experienced intensity of the negative images presented in this task increased when the task was repeated a second time, but for the participants who trained socio-affective skills during the Affect module this sensitization effect was buffered. These results suggest that already after 3 months, the compassion-based Affect module decreased the experience of negative affect when exposed to negative social stimuli and thus helped buffer against sensitization effects to the task when repeated a second time. After 9 months of training, the participants developed better regulation of negative emotions whenever they trained socio-affective skills, as shown by the decrease in rated negative affect after the overall training as well as after Affect vs. retest (averaged from $\mathrm{T} 0$ to $\mathrm{T} 3$ ). This significant training-related behavioural reduction of reported negative affect after compassion-based training was also reflected on a neuronal level by increased activation in the right SMG. Notably, the brain activation pattern observed after the Affect modules when participants are exposed to pictures depicting people suffering, partially overlaps with the empathy network defined in the same participants with another task, the socalled EmpaToM, at baseline (Kanske et al., 2015) (Figure S4a) and is consistent with findings from meta-analyses on empathy studies (Bzdok et al., 2012; Fan et al., 2011; Lamm et al., 2011). This finding is also in accordance with previous ReSource project related findings from our group, that demonstrated increased cortical thickness in socio-emotional networks related to empathy and compassion (mid-insula and SMG) after participants underwent the Affect module of the ReSource project (Valk et al., 2017). In the compassion network, the SMG might be important for supporting the ability to engage in self-other distinction, a process needed for 
example if wanting to overcome emotional egocentric bias (Silani et al., 2013; Steinbeis et al., 2014). Indeed, studies showed that participants tended to make inaccurate empathic judgements about others when their own affective states were incongruent to the one of another and after disruption of the right SMG with transcranial magnetic stimulation (Silani et al., 2013). Furthermore, increased connectivity between the SMG and the dorso-lateral PFC in children predicted lesser egocentric bias in the affective domain (Steinbeis et al., 2014). Even though not significant after correction, the increased activation of the ventrolateral PFC is in line with this previous research and might be related to better emotion regulation after training Affect. Indeed, lateral regions of the PFC, are known to be involved in cognitive control, reappraisal and inhibitory processes (Aron et al., 2003; Grecucci et al., 2013; Hampshire et al., 2010).

Interestingly, and contrary to our hypotheses and previous ReSource project findings showing increased compassion related ratings and brain plasticity after the Affect module (Trautwein et al., 2020; Valk et al., 2017), we did not observe increased activation in the regions classically involved in compassion, care and gratitude, such as the OFC, the VS or the VTA (Engen and Singer, 2015; Klimecki et al., 2013; Klimecki et al., 2014) after training the Affect module. One explanation could be the nature of the task used here, which involved natural processing of emotion when being exposed to emotional stimuli. In contrast to some previous studies on compassion-based meditation training (Engen and Singer, 2015; Klimecki et al., 2013; Klimecki et al., 2014; Leung et al., 2018; Weng et al., 2013; Weng et al., 2018), we neither explicitly instructed the participants to use specific emotion regulation strategies, such as the generation of positive affect or loving-kindness meditation, nor asked for empathy or compassion ratings (Trautwein et al., 2020). Therefore, the participants may have used other emotion regulation capacities during the task, e.g. accepting and regulating difficult negative emotions when these arise, rather than actively generating positive affect of compassion and concern. Of note, the Affect module also included daily 10-minutes Dyadic practices with a 
partner (Kok and Singer, 2017) where participants trained to report difficult emotions experienced during their day and to accept them without judging these. Such daily practice may have helped participants to learn acceptance and non-reactivity towards experienced negative affect.

Relative to the RCC group, we also observed increased positive ratings for positive stimuli after the overall 9-month training, which seem to be driven by the change after the Perspective training module (Figure 2, Panels c and d and Figure S3, Panel b). The overall increase of positive ratings after the 9-months training was associated with increased activity of occipito-temporal regions involved in the processing of positive vs. neutral stimuli at T0, the lingual region of the occipital cortex in particular. As we did not expect a change in functional activation in visual regions after the training this result is difficult to explain. However, it might be linked to increased visual processing of positively valenced stimuli, similar of that observed for negative high arousing stimuli (Lang et al., 1998; Vuilleumier, 2005; Vuilleumier and Pourtois, 2007). Positive ratings seemed to increase especially after Perspective training, which aims at improving metacognitive skills and perspective taking on ones' own and others' thoughts and believes. The training of socio-cognitive skills could then allow people to better understand and share positive emotions. This argument is supported by an observed trend for increased activity after Perspective in two regions reliably associated with ToM, the middle temporal gyrus and the dorso-medial PFC (Figure S4b). Although these results are exploratory, the location of change in brain activation partially overlaps with the ToM network identified with the EmpaToM task at baseline (Kanske et al., 2015).

The first limitation inherent to this study is that it is impossible to conduct a doubleblind RCT in such mental training and meditation-based studies. Without revealing the objectives of the experimental task, the participants obviously know what type of mental training they carry out when practicing. Indeed, they have to be instructed in these practices to 
be able to consciously and internally perform them later on. Thus, the instructions given during each module may have created a demand effect. However, given the implicit nature of the task used, it is unlikely that the participants were able to explicitly control their emotional and brain response to adapt to these demands. In fact, no instructions were given to the participants, so any regulation strategies employed were implicit and spontaneous, thus likely an actual outcome of the training. Furthermore, each module consisted of many different mental practices belonging to more general categories and thus it was not possible to guess which task would assess which specific effect of the respective practice in a module.

In that context, the fact that each mental training module in the ReSource project includes multiple exercises (such as loving-meditation and affective dyads in the Affect module) may also be seen as a second limitation. Indeed, is difficult to isolate the specific effect of each exercise on emotional reactivity. However, in comparison to previous studies focusing on 8week programs such as MBSR, MBCT and compassion-based intervention (Gilbert, 2009; Jazaieri et al., 2013; Kabat-Zinn, 2003; Neff and Germer, 2013; Williams et al., 2014), the ReSource project allowed a systematic comparison of classes of mental training practices, while including active control groups and large samples. A recent review on ReSource project findings from our group (Singer and Engert, 2019), revealed many differential effects of practice types on all levels: the level of subjective experience, behaviour, brain plasticity and stress-reduction. Accordingly, we could show here that socio-affective and compassion-based practices are specifically efficient to reduce negative emotional reactivity.

In conclusion, being confronted with the suffering of others can be a potent source of personal distress and may have deleterious mental health effects. This is seen in the high stress levels and burnout rates often reported for professionals tasked with caring for suffering individuals, such as physicians (Shanafelt et al., 2012) and nurses (Adriaenssens et al., 2015). Decreasing negative affect and increasing positive one is particularly important in clinical 
settings. The applications of meditation-based mental training could thus promote resilience for exposure to others' suffering (Klimecki and Singer, 2012). Our results revealed that the 9month mental training program of the ReSource project with its three 3-month training modules, and here especially the compassion-based Affect module lead to a decrease of experienced negative affect when confronted with emotionally distressing social stimuli and that this was associated with changes in functional plasticity in brain networks playing a key role in emotion regulation. Given the importance of affective processes on both a social and clinical level, the impact of compassion-based socio-affective mental training for a) people suffering from mental disorders, b) for health-workers such as nurses or medical doctors as well as c) for children, teachers and educators, should be further explore. 


\section{Funding}

This study forms part of the ReSource Project, headed by Tania Singer. Data for this project were collected between 2013 and 2016 at the former department of Social Neuroscience at the Max Planck Institute for Human Cognitive and Brain Sciences Leipzig. Tania Singer (Principal Investigator) received funding for the ReSource Project from the European Research Council under the European Community's Seventh Framework Program (FP7/20072013) ERC Grant Agreement Number 205557.

\section{Acknowledgments}

We are thankful to the members of the Social Neuroscience Department involved in the ReSource Project over many years, in particular to the teachers of the ReSource intervention program, to Astrid Ackermann, Christina Bochow, Matthias Bolz and Sandra Zurborg for managing the large-scale longitudinal study, to Elisabeth Murzik, Sylvia Tydecks, Kerstin Träger, and Nadine Otto for help with recruiting and data archiving, to Henrik Grunert for technical assistance, to Manuela Hofmann, Sylvie Neubert, and Nicole Pampus for help with data collection, and to Hannes Niederhausen and Torsten Kästner for data management.

\section{Author contributions}

T.S. initiated and developed the ReSource Project and model as well as the training protocol and secured all funding. T.S., P.K. and H.E. contributed to the present study design and development of the task. P.K. and H.E. were involved in testing and data collection. P.F wrote de first draft and performed the data analysis and interpretation under the supervision of P.K. All authors contributed to writing up or revising the paper and approved the final version of the manuscript for submission.

\section{Competing interests}

The authors declare no competing financial interest. 


\section{Data Availability}

In line with new data regulations (General Data Protection Regulation, GDPR), we regret that our data cannot be shared publicly because we did not obtain explicit participant agreement for data-sharing with parties outside the Max Planck Institute for Human Cognitive and Brain Sciences (MPI CBS). The present work is based on personal data (age, sex and medical data) that could be matched to individuals. The data is therefore pseudonymized rather than anonymized and falls under the GDPR. Data are available upon request (contact via corresponding author email address).

\section{Code Availability}

The code that supports the findings of this study is available from the corresponding author upon request. 


\section{References}

Adriaenssens, J., De Gucht, V., Maes, S., 2015. Causes and consequences of occupational stress in emergency nurses, a longitudinal study. Journal of Nursing Management 23, 346-358.

Allen, M., Dietz, M., Blair, K.S., van Beek, M., Rees, G., Vestergaard-Poulsen, P., Lutz, A., Roepstorff, A., 2012. Cognitive-affective neural plasticity following active-controlled mindfulness intervention. Journal of Neuroscience 32, 15601-15610.

Aron, A.R., Fletcher, P.C., Bullmore, E.T., Sahakian, B.J., Robbins, T.W., 2003. Stop-signal inhibition disrupted by damage to right inferior frontal gyrus in humans. Nature neuroscience $6,115-116$.

Ashburner, J., 2007. A fast diffeomorphic image registration algorithm. NeuroImage 38, 95113.

Bates, D., Mächler, M., Bolker, B., Walker, S., 2015. Fitting linear mixed-effects models using lme4. Journal of Statistical Software 67.

Baucom, L.B., Wedell, D.H., Wang, J., Blitzer, D.N., Shinkareva, S.V., 2012. Decoding the neural representation of affective states. NeuroImage 59, 718-727.

Buckner, R.L., Andrews-Hanna, J.R., Schacter, D.L., 2008. The brain's default network: anatomy, function, and relevance to disease Annals of the New York Academy of Sciences $1124,1-38$.

Buhle, J.T., Silvers, J.A., Wager, T.D., Lopez, R., Onyemekwu, C., Kober, H., Weber, J., Ochsner, K.N., 2014. Cognitive reappraisal of emotion: a meta-analysis of human neuroimaging studies. Cerebral Cortex 24, 2981-2990.

Burgdorf, J., Panksepp, J., 2006. The neurobiology of positive emotions. Neuroscience \& Biobehavioral Reviews 30, 173-187. 
Bzdok, D., Schilbach, L., Vogeley, K., Schneider, K., Laird, A.R., Langner, R., Eickhoff, S.B., 2012. Parsing the neural correlates of moral cognition: ALE meta-analysis on morality, theory of mind, and empathy. Brain Structure and Function 217, 783-796.

Carter, C., Keverne, E.B., 2002. The neurobiology of social affiliation and pair bonding. Hormones, brain and behavior. Elsevier, pp. 299-337.

Chiesa, A., Serretti, A., Jakobsen, J.C., 2013. Mindfulness: Top-down or bottom-up emotion regulation strategy? Clinical Psychology Review 33, 82-96.

Creswell, J.D., Pacilio, L.E., Lindsay, E.K., Brown, K.W., 2014. Brief mindfulness meditation training alters psychological and neuroendocrine responses to social evaluative stress. Psychoneuroendocrinology 44, 1-12.

Dan-Glauser, E.S., Scherer, K.R., 2011. The Geneva affective picture database (GAPED): a new 730-picture database focusing on valence and normative significance. Behavior research methods 43, 468.

De Vignemont, F., Singer, T., 2006. The empathic brain: how, when and why? Trends in cognitive sciences 10, 435-441.

Desbordes, G., Negi, L.T., Pace, T.W., Wallace, B.A., Raison, C.L., Schwartz, E.L., 2012. Effects of mindful-attention and compassion meditation training on amygdala response to emotional stimuli in an ordinary, non-meditative state. Frontiers in human neuroscience 6, 292. Engen, H.G., Kanske, P., Singer, T., 2017. The neural component-process architecture of endogenously generated emotion. Social Cognitive and Affective Neuroscience 12, 197-211. Engen, H.G., Singer, T., 2015. Compassion-based emotion regulation up-regulates experienced positive affect and associated neural networks. Social Cognitive and Affective Neuroscience $10,1291-1301$. 
Fan, Y., Duncan, N.W., de Greck, M., Northoff, G., 2011. Is there a core neural network in empathy? An fMRI based quantitative meta-analysis. Neuroscience \& Biobehavioral Reviews $35,903-911$.

Farb, N.A., Anderson, A.K., Mayberg, H., Bean, J., McKeon, D., Segal, Z.V., 2010. Minding one's emotions: mindfulness training alters the neural expression of sadness. Emotion 10, 2533.

Farb, N.A., Anderson, A.K., Segal, Z.V., 2012. The mindful brain and emotion regulation in mood disorders. The Canadian Journal of Psychiatry 57, 70-77.

Farb, N.A., Segal, Z.V., Mayberg, H., Bean, J., McKeon, D., Fatima, Z., Anderson, A.K., 2007. Attending to the present: mindfulness meditation reveals distinct neural modes of selfreference. Social Cognitive and Affective Neuroscience 2, 313-322.

Feldman Barrett, L., Russell, J.A., 1998. Independence and bipolarity in the structure of current affect. Journal of personality and social psychology 74, 967.

Fox, K.C., Dixon, M.L., Nijeboer, S., Girn, M., Floman, J.L., Lifshitz, M., Ellamil, M., Sedlmeier, P., Christoff, K., 2016. Functional neuroanatomy of meditation: A review and metaanalysis of 78 functional neuroimaging investigations. Neuroscience \& Biobehavioral Reviews $65,208-228$.

Fox, K.C., Nijeboer, S., Dixon, M.L., Floman, J.L., Ellamil, M., Rumak, S.P., Sedlmeier, P., Christoff, K., 2014. Is meditation associated with altered brain structure? A systematic review and meta-analysis of morphometric neuroimaging in meditation practitioners. Neuroscience $\&$ Biobehavioral Reviews 43, 48-73.

Frith, C., Frith, U., 2005. Theory of mind. Current Biology 15, R644-R645.

Froeliger, B., Garland, E.L., Modlin, L.A., McClernon, F.J., 2012. Neurocognitive correlates of the effects of yoga meditation practice on emotion and cognition: a pilot study. Frontiers in integrative neuroscience 6,48 . 
Gilbert, P., 2009. Introducing compassion-focused therapy. Advances in psychiatric treatment $15,199-208$.

Gilbert, P., 2017. Compassion: Concepts, research and applications. Taylor \& Francis.

Goldin, P., Ziv, M., Jazaieri, H., Gross, J., 2012. Randomized controlled trial of mindfulnessbased stress reduction versus aerobic exercise: effects on the self-referential brain network in social anxiety disorder. Frontiers in human neuroscience 6, 295.

Goldin, P.R., Gross, J.J., 2010. Effects of mindfulness-based stress reduction (MBSR) on emotion regulation in social anxiety disorder. Emotion 10, 83.

Grecucci, A., Giorgetta, C., Bonini, N., Sanfey, A.G., 2013. Reappraising social emotions: the role of inferior frontal gyrus, temporo-parietal junction and insula in interpersonal emotion regulation. Frontiers in human neuroscience 7.

Gu, J., Strauss, C., Bond, R., Cavanagh, K., 2015. How do mindfulness-based cognitive therapy and mindfulness-based stress reduction improve mental health and wellbeing? A systematic review and meta-analysis of mediation studies. Clinical Psychology Review 37, 1-12.

Guendelman, S., Medeiros, S., Rampes, H., 2017. Mindfulness and emotion regulation: Insights from neurobiological, psychological, and clinical studies. Frontiers in psychology 8, 220.

Guillaume, B., Hua, X., Thompson, P.M., Waldorp, L., Nichols, T.E., Initiative, A.s.D.N., 2014. Fast and accurate modelling of longitudinal and repeated measures neuroimaging data. NeuroImage 94, 287-302.

Hampshire, A., Chamberlain, S.R., Monti, M.M., Duncan, J., Owen, A.M., 2010. The role of the right inferior frontal gyrus: inhibition and attentional control. NeuroImage 50, 1313-1319. Heeren, A., Philippot, P., 2011. Changes in ruminative thinking mediate the clinical benefits of mindfulness: Preliminary findings. Mindfulness 2, 8-13. 
Hölzel, B.K., Hoge, E.A., Greve, D.N., Gard, T., Creswell, J.D., Brown, K.W., Barrett, L.F., Schwartz, C., Vaitl, D., Lazar, S.W., 2013. Neural mechanisms of symptom improvements in generalized anxiety disorder following mindfulness training. NeuroImage: Clinical 2, 448-458. Jazaieri, H., Jinpa, G.T., McGonigal, K., Rosenberg, E.L., Finkelstein, J., Simon-Thomas, E., Cullen, M., Doty, J.R., Gross, J.J., Goldin, P.R., 2013. Enhancing compassion: A randomized controlled trial of a compassion cultivation training program. Journal of Happiness Studies 14, $1113-1126$.

Johnson, D.C., Thom, N.J., Stanley, E.A., Haase, L., Simmons, A.N., Shih, P.-a.B., Thompson, W.K., Potterat, E.G., Minor, T.R., Paulus, M.P., 2014. Modifying resilience mechanisms in atrisk individuals: a controlled study of mindfulness training in Marines preparing for deployment. American Journal of Psychiatry 171, 844-853.

Kabat-Zinn, J., 2003. Mindfulness-based interventions in context: past, present, and future. Clinical psychology: Science and practice 10, 144-156.

Kanske, P., Böckler, A., Trautwein, F.-M., Parianen Lesemann, F.H., Singer, T., 2016. Are strong empathizers better mentalizers? Evidence for independence and interaction between the routes of social cognition. Social Cognitive and Affective Neuroscience 11, 1383-1392.

Kanske, P., Böckler, A., Trautwein, F.-M., Singer, T., 2015. Dissecting the social brain: Introducing the EmpaToM to reveal distinct neural networks and brain-behavior relations for empathy and Theory of Mind. NeuroImage 122, 6-19.

Klimecki, O., Singer, T., 2012. Empathic distress fatigue rather than compassion fatigue? Integrating findings from empathy research in psychology and social neuroscience. Pathological altruism, 368-383.

Klimecki, O.M., Leiberg, S., Lamm, C., Singer, T., 2013. Functional neural plasticity and associated changes in positive affect after compassion training. Cerebral Cortex 23, 1552-1561. 
Klimecki, O.M., Leiberg, S., Ricard, M., Singer, T., 2014. Differential pattern of functional brain plasticity after compassion and empathy training. Social Cognitive and Affective Neuroscience 9, 873-879.

Kok, B.E., Singer, T., 2017. Effects of contemplative dyads on engagement and perceived social connectedness over 9 months of mental training: A randomized clinical trial. JAMA Psychiatry 74, 126-134.

Kragel, P.A., LaBar, K.S., 2016. Decoding the nature of emotion in the brain. Trends in cognitive sciences $20,444-455$.

Kral, T.R., Schuyler, B.S., Mumford, J.A., Rosenkranz, M.A., Lutz, A., Davidson, R.J., 2018. Impact of short-and long-term mindfulness meditation training on amygdala reactivity to emotional stimuli. NeuroImage 181, 301-313.

Kuyken, W., Warren, F.C., Taylor, R.S., Whalley, B., Crane, C., Bondolfi, G., Hayes, R., Huijbers, M., Ma, H., Schweizer, S., 2016. Efficacy of mindfulness-based cognitive therapy in prevention of depressive relapse: An individual patient data meta-analysis from randomized trials. JAMA Psychiatry 73, 565-574.

Lamm, C., Decety, J., Singer, T., 2011. Meta-analytic evidence for common and distinct neural networks associated with directly experienced pain and empathy for pain. NeuroImage 54, 2492-2502.

Lang, P.J., Bradley, M.M., Cuthbert, B.N., 1999. International affective picture system (IAPS): Technical manual and affective ratings. Gainesville, FL: The Center for Research in Psychophysiology, University of Florida.

Lang, P.J., Bradley, M.M., Fitzsimmons, J.R., Cuthbert, B.N., Scott, J.D., Moulder, B., Nangia, V., 1998. Emotional arousal and activation of the visual cortex: an fMRI analysis. Psychophysiology 35, 199-210. 
Lee, T.M., Leung, M.-K., Hou, W.-K., Tang, J.C., Yin, J., So, K.-F., Lee, C.-F., Chan, C.C., 2012. Distinct neural activity associated with focused-attention meditation and loving-kindness meditation. PLOS ONE 7.

Leung, M.-K., Lau, W.K., Chan, C.C., Wong, S.S., Fung, A.L., Lee, T.M., 2018. Meditationinduced neuroplastic changes in amygdala activity during negative affective processing. Social neuroscience $13,277-288$.

Lindquist, K.A., Wager, T.D., Kober, H., Bliss-Moreau, E., Barrett, L.F., 2011. The brain basis of emotion: A meta-analytic review. Behavioral and Brain Sciences 173, 1-86.

Lindquist, K.A., Wager, T.D., Kober, H., Bliss-Moreau, E., Barrett, L.F., 2012. The brain basis of emotion: a meta-analytic review. The Behavioral and brain sciences 35, 121 .

Lutz, A., Brefczynski-Lewis, J., Johnstone, T., Davidson, R.J., 2008. Regulation of the neural circuitry of emotion by compassion meditation: effects of meditative expertise. PLOS ONE 3, e1897.

Lutz, A., Greischar, L.L., Perlman, D.M., Davidson, R.J., 2009. BOLD signal in insula is differentially related to cardiac function during compassion meditation in experts vs. novices. NeuroImage 47, 1038-1046.

Lutz, J., Herwig, U., Opialla, S., Hittmeyer, A., Jäncke, L., Rufer, M., Holtforth, M.G., Brühl, A.B., 2014. Mindfulness and emotion regulation-an fMRI study. Social Cognitive and Affective Neuroscience 9, 776-785.

Magalhaes, A.A., Oliveira, L., Pereira, M.G., Menezes, C.B., 2018. Does meditation alter brain responses to negative stimuli? a systematic review. Frontiers in human neuroscience 12, 448. McCall, C., Singer, T., 2012. The animal and human neuroendocrinology of social cognition, motivation and behavior. Nature neuroscience 15, 681-688.

Neff, K.D., Germer, C.K., 2013. A pilot study and randomized controlled trial of the mindful self-compassion program. Journal of Clinical Psychology 69, 28-44. 
O’Doherty, J.P., 2004. Reward representations and reward-related learning in the human brain: insights from neuroimaging. Current opinion in neurobiology 14, 769-776.

Ochsner, K.N., Bunge, S.A., Gross, J.J., Gabrieli, J.D.E., 2002. Rethinking feelings: An fMRI study of the cognitive regulation of emotion. Journal of Cognitive Neuroscience 14, 1215-1229. Ochsner, K.N., Gross, J.J., 2005. The cognitive control of emotion. Trends in cognitive sciences 9, 242-249.

Pace, T.W., Negi, L.T., Adame, D.D., Cole, S.P., Sivilli, T.I., Brown, T.D., Issa, M.J., Raison, C.L., 2009. Effect of compassion meditation on neuroendocrine, innate immune and behavioral responses to psychosocial stress. Psychoneuroendocrinology 34, 87-98.

Phan, K.L., Wager, T., Taylor, S.F., Liberzon, I., 2002. Functional neuroanatomy of emotion: a meta-analysis of emotion activation studies in PET and fMRI. NeuroImage 16, 331-348.

Phelps, E.A., LeDoux, J.E., 2005. Contributions of the amygdala to emotion processing: from animal models to human behavior. Neuron 48, 175-187.

Piet, J., Hougaard, E., 2011. The effect of mindfulness-based cognitive therapy for prevention of relapse in recurrent major depressive disorder: a systematic review and meta-analysis. Clinical Psychology Review 31, 1032-1040.

Preckel, K., Trautwein, F.-M., Paulus, F.M., Kirsch, P., Krach, S., Singer, T., Kanske, P., 2019. Neural mechanisms of affective matching across faces and scenes. Scientific reports 9, 1-10. Premack, D., Woodruff, G., 1978. Does the chimpanzee have a theory of mind? Behavioral and Brain Sciences 1, 515-526.

Saxe, R., Kanwisher, N., 2003. People thinking about thinking people: the role of the temporoparietal junction in "theory of mind". NeuroImage 19, 1835-1842.

Schultz, W., 2006. Behavioral theories and the neurophysiology of reward. Annu. Rev. Psychol. $57,87-115$. 
Schurz, M., Radua, J., Aichhorn, M., Richlan, F., Perner, J., 2014. Fractionating theory of mind: A meta-analysis of functional brain imaging studies. Neuroscience \& Biobehavioral Reviews 42, 9-34.

Sedlmeier, P., Eberth, J., Schwarz, M., Zimmermann, D., Haarig, F., Jaeger, S., Kunze, S., 2012. The psychological effects of meditation: a meta-analysis. Psychological bulletin 138, 1139.

Shanafelt, T.D., Boone, S., Tan, L., Dyrbye, L.N., Sotile, W., Satele, D., West, C.P., Sloan, J., Oreskovich, M.R., 2012. Burnout and satisfaction with work-life balance among US physicians relative to the general US population. Archives of internal medicine 172, 1377-1385.

Silani, G., Lamm, C., Ruff, C.C., Singer, T., 2013. Right supramarginal gyrus is crucial to overcome emotional egocentricity bias in social judgments. Journal of Neuroscience 33, 1546615476.

Singer, T., 2012. The past, present and future of social neuroscience: a European perspective. NeuroImage 61, 437-449.

Singer, T., Engert, V., 2019. It matters what you practice: Differential training effects on subjective experience, behavior, brain and body in the ReSource Project. Current opinion in psychology 28, 151-158.

Singer, T., Klimecki, O.M., 2014. Empathy and compassion. Current Biology 24, R875-R878. Singer, T., Kok, B.E., Bornemann, B., Zurborg, S., Bolz, M., Bochow, C., 2016. The ReSource Project: Background, design, samples, and measurements (2nd ed.). Max Planck Institute for Human Cognition and Brain Science, Leipzig, Germany.

Singer, T., Seymour, B., O'Doherty, J., Kaube, H., Dolan, R.J., Frith, C.D., 2004. Empathy for pain involves the affective but not sensory components of pain. Science 303, 1157-1162. 
Somerville, L.H., Wagner, D.D., Wig, G.S., Moran, J.M., Whalen, P.J., Kelley, W.M., 2012. Interactions between transient and sustained neural signals support the generation and regulation of anxious emotion. Cerebral Cortex 23, 49-60.

Steinbeis, N., Bernhardt, B.C., Singer, T., 2014. Age-related differences in function and structure of rSMG and reduced functional connectivity with DLPFC explains heightened emotional egocentricity bias in childhood. Social Cognitive and Affective Neuroscience 10, $302-310$

Tang, Y.-Y., Hölzel, B.K., Posner, M.I., 2015. The neuroscience of mindfulness meditation. Nature Reviews Neuroscience 16, 213-225.

Taylor, V.A., Grant, J., Daneault, V., Scavone, G., Breton, E., Roffe-Vidal, S., Courtemanche, J., Lavarenne, A.S., Beauregard, M., 2011. Impact of mindfulness on the neural responses to emotional pictures in experienced and beginner meditators. NeuroImage 57, 1524-1533.

Teasdale, J.D., Moore, R.G., Hayhurst, H., Pope, M., Williams, S., Segal, Z.V., 2002. Metacognitive awareness and prevention of relapse in depression: empirical evidence. Journal of Consulting and Clinical Psychology 70, 275.

Trautwein, F.-M., Kanske, P., Böckler, A., Singer, T., 2020. Differential benefits of mental training types for attention, compassion, and theory of mind. Cognition 194, 104039.

Tzourio-Mazoyer, N., Landeau, B., Papathanassiou, D., Crivello, F., Etard, O., Delcroix, N., Mazoyer, B., Joliot, M., 2002. Automated anatomical labeling of activations in SPM using a macroscopic anatomical parcellation of the MNI MRI single-subject brain. NeuroImage 15, 273-289.

Valk, S.L., Bernhardt, B.C., Trautwein, F.-M., Böckler, A., Kanske, P., Guizard, N., Collins, D.L., Singer, T., 2017. Structural plasticity of the social brain: Differential change after socioaffective and cognitive mental training. Science Advances 3, e1700489. 
Vuilleumier, P., 2005. How brains beware: neural mechanisms of emotional attention. Trends in cognitive sciences $9,585-594$.

Vuilleumier, P., Pourtois, G., 2007. Distributed and interactive brain mechanisms during emotion face perception: evidence from functional neuroimaging. Neuropsychologia 45, 174194.

Wallace, B.A., Shapiro, S.L., 2006. Mental balance and well-being: building bridges between Buddhism and Western psychology. American Psychologist 61, 690.

Weng, H.Y., Fox, A.S., Shackman, A.J., Stodola, D.E., Caldwell, J.Z., Olson, M.C., Rogers, G.M., Davidson, R.J., 2013. Compassion training alters altruism and neural responses to suffering. Psychological science 24, 1171-1180.

Weng, H.Y., Lapate, R.C., Stodola, D.E., Rogers, G.M., Davidson, R.J., 2018. Visual attention to suffering after compassion training is associated with decreased amygdala responses. Frontiers in psychology 9, 771.

Wessa, M., Kanske, P., Neumeister, P., Bode, K., Heissler, J., Schönfelder, S., 2010. EmoPicS: subjective and psychophysiological evaluation of new imagery for clinical biopsychological research. Z. Klin. Psychol. Psychother. Suppl 1, 11-77.

Williams, J.M.G., Crane, C., Barnhofer, T., Brennan, K., Duggan, D.S., Fennell, M.J., Hackmann, A., Krusche, A., Muse, K., Von Rohr, I.R., 2014. Mindfulness-based cognitive therapy for preventing relapse in recurrent depression: a randomized dismantling trial. Journal of Consulting and Clinical Psychology 82, 275.

Young, K.S., van der Velden, A.M., Craske, M.G., Pallesen, K.J., Fjorback, L., Roepstorff, A., Parsons, C.E., 2018. The impact of mindfulness-based interventions on brain activity: A systematic review of functional magnetic resonance imaging studies. Neuroscience \& Biobehavioral Reviews 84, 424-433. 
Zeidan, F., Emerson, N.M., Farris, S.R., Ray, J.N., Jung, Y., McHaffie, J.G., Coghill, R.C., 2015. Mindfulness meditation-based pain relief employs different neural mechanisms than placebo and sham mindfulness meditation-induced analgesia. Journal of Neuroscience 35, $15307-15325$. 